\title{
Extracorporeal membrane oxygenation for the treatment of severe refractory hantavirus cardiopulmonary syndrome
}

\author{
Han Yao MD Msc, Emily G. McDonald MD MSc
}

Competing interests: None declared.

This article has been peer reviewed.

The authors have obtained patient consent.

Correspondence to: Han Yao, han.yao@mail.mcgill. ca

CMAJ 2016. DOI:10.1503 /cmaj.160397
A previously healthy 22-year-old man presented to a community hospital with diarrhea, vomiting, and a fever of $40^{\circ} \mathrm{C}$ that had lasted for two days. He was admitted to the intensive care unit.

Initially, the patient's cardiopulmonary findings were normal. He had a soft but tender abdomen, and musculoskeletal and skin examinations were normal. Soon after initial fever onset, rapid hemodynamic and respiratory failure developed. Within 18 hours of presentation, the patient had become profoundly hypoxemic and required intubation and ventilator support. During the same time, progressive hemodynamic instability developed (blood pressure 85/65 $\mathrm{mm} \mathrm{Hg}$ ) despite five litres of lactated Ringer solution and incremental doses of vasopressors administered intravenously.

Initial laboratory investigations showed elevated hemoglobin (180 [normal 135-175] g/L), hematocrit $63 \%$ (normal 42\%-52\%), thrombocytopenia $(50$ [normal $\left.140-450] \times 10^{9} / \mathrm{L}\right)$, normal white blood cell count $(7.5$ [normal $4.5-$ $\left.11.00] \times 10^{9} / \mathrm{L}\right)$ with a normal differential, normal liver enzymes and normal coagulation parameters. Ceftriaxone and azithromycin were administered for presumed sepsis from community-acquired pneumonia, and the patient was

\section{KEY POINTS}

- A travel history, including exposure to rodents or travel to a Hantavirus endemic area, should be obtained in cases of unexplained acute respiratory distress syndrome with hemoconcentration.

- In patients with suspected hantavirus cardiopulmonary syndrome, the triad of thrombocytopenia, left-shifted granulocytic cell line and immunoblasts on peripheral blood film may help to establish the diagnosis presumptively.

- Serologic testing for serum antihantavirus immunoglobulins $M$ and $G$ is the preferred diagnostic modality for hantavirus cardiopulmonary syndrome; polymerase chain reaction testing is less sensitive and should only be used as a confirmatory test or to identify potential sites of infection.

- Rapid and early transfer to a centre capable of extracorporeal membrane oxygenation should be considered when hantavirus cardiopulmonary syndrome is suspected, along with the following criteria: cardiac index less than $2.0 \mathrm{~L} / \mathrm{min}$ per $\mathrm{m}^{2}$ and any of refractory shock, severe hypoxia or need for external cardiac massage. urgently transferred to a tertiary care centre.

The patient's cardiopulmonary status continued to deteriorate despite controlled ventilation using pressure-regulated volume control mode; his tidal volume was $4.6 \mathrm{~mL} / \mathrm{kg}$ (peak plateau pressure 22 $\mathrm{mm} \mathrm{H}_{2} \mathrm{O}$ ), his positive end-expiratory pressure was $18 \mathrm{~cm} \mathrm{H}_{2} \mathrm{O}$, his fractional inspired oxygen ( $\left.\mathrm{FiO} 2\right)$ was $100 \%$, and neuromuscular blockade with rocuronium was administered. Arterial partial pressure of oxygen $\left(\mathrm{PaO}_{2}\right)$ was $52.8 \mathrm{~mm} \mathrm{Hg}$, with a $\mathrm{PaO}_{2} / \mathrm{FiO}_{2}$ ratio of 52.8 , indicating severe hypoxemia. Inhaled nitric oxide was administered without improvement.

The patient's central venous pressure was low $(1 \mathrm{~mm} \mathrm{Hg}$ ), his cardiac index was 1.6 (normal 2.8-4.2) $\mathrm{L} / \mathrm{min}$ per $\mathrm{m}^{2}$ and his arterial lactate level was 4.1 (normal 0-2) $\mathrm{mmol} / \mathrm{L}$ despite three additional litres of crystalloid and albumin infusions and maximal doses of vasopressor therapy.

The patient's white blood cell count rose to 53.4 $\times 10^{\circ} / \mathrm{L}$, and a peripheral blood film showed leukocytosis with left shift, thrombocytopenia and immunoblasts. Hemoconcentration was evident, with a peak hematocrit of $64 \%$. Disseminated intravascular coagulopathy developed with an international normalized ratio of 1.93 (normal 0.9-1.1) and partial thromboplastin time of 60.3 (normal 31.643.5) seconds. A radiograph of the chest showed extensive bilateral airspace disease (Figure 1).

Antimicrobial coverage was broadened to include meropenem and vancomycin. A differential diagnosis of primary respiratory infection, such as legionella pneumonia, was entertained. Clindamycin and immunoglobulins were given intravenously for the possibility of toxic shock syndrome. Acute decompensated heart failure due to viral myocarditis and acute valvulopathy secondary to infective endocarditis were also considered. An urgent transesophageal echocardiogram performed bedside showed mildly decreased ejection fraction $(45 \%-50 \%)$ without associated valvulopathy or vegetation. Noninfectious diseases such as granulomatosis with polyangiitis were thought to be less likely, and steroids were not administered. Four sets of blood cultures, a stool culture for bacterial 
pathogens and a nasopharyngeal swab for respiratory viruses had negative results.

Given the severity of the patient's clinical status, venoarterial extracorporeal membrane oxygenation (VA-ECMO) support was started eight hours after transfer (Appendix 1, available at www.cmaj.ca/ lookup/suppl/doi:10.1503/cmaj.160397/-/DC1 for further details). Oxygenation and perfusion parameters normalized. Renal failure subsequently developed, with peak creatinine of 328 (normal 55-110) $\mu \mathrm{mol} / \mathrm{L}$, thereby necessitating continuous renal replacement therapy for 48 hours.

Within 48 hours, cardiac function improved and VA-ECMO was withdrawn after 112 hours. Upon further questioning, family members reported that the patient had been on a military training exercise in Wainwright, Alberta for about five weeks. Mice were present under platforms where the soldiers slept at night, and exposure to aerosolized soil occurred through various military exercises. Based on exposure history, hemoconcentration and rapid cardiopulmonary collapse, Hantavirus serologies and nasopharyngeal swab samples for Hantavirus polymerase chain reaction (PCR) analysis were sent. Qualitative serologic testing for antihantavirus immunoglobulins $\mathrm{M}$ and $\mathrm{G}$ (IgM and $\mathrm{IgG}$ ) showed positive results. These results were later confirmed by a positive PCR test for Sin Nombre virus.

\section{Discussion}

Hantaviruses are single-stranded negative-sense RNA viruses of the Bunyaviridae family. At least 11 species are associated with human disease, with transmission occurring through the inhalation of aerosolized viral particles from urine, feces or other secretions of infected rodents. ${ }^{1}$ In humans, two major forms of disease exist: hemorrhagic fever with renal syndrome and hantavirus cardiopulmonary syndrome. Hantavirus cardiopulmonary syndrome was first described in 1993 in association with infections with the Sin Nombre virus, a member of the Hantavirus genus. Sin Nombre virus is carried by the deer mouse, Peromyscus maniculatus, and is the leading cause of hantavirus cardiopulmonary syndrome in North America. Although Sin Nombre virus is limited to North America, hantavirus cardiopulmonary syndrome caused by other species of Hantavirus has been identified throughout Northern, Central and South America. ${ }^{1}$

\section{North American epidemiology}

The Canada Communicable Disease Report on hantavirus cardiopulmonary syndrome provides epidemiological data of annual incidence from 1989 to 2014. ${ }^{2}$ As of Dec. 31, 2014, a total of 109 laboratory-confirmed cases of the condition were identified in Canada, and more than 600 cases were identified in the United States. ${ }^{2}$ Canadian data show a male predominance (67\%), mean age of 40 years, peak incidence in spring and early summer and mortality of $29 \% .^{2}$ In recent years, there has been an increase in the number of cases of hantavirus cardiopulmonary syndrome in Canada, from a mean of 4 to 5 cases per year from 2006-2012 to 13 and 10 cases in 2013 and 2014, respectively. ${ }^{2}$ Most cases occur in western Canada, and a case series involving 19 patients from Northern Alberta previously reported a mortality of $26 \%{ }^{3}$

\section{Human disease}

The incubation period from infection to presentation with cardiopulmonary syndrome can last up to 42 days. The median incubation periods were 30.5 (range 20-49) days in a Yosemite outbreak of hantavirus cardiopulmonary syndrome caused by Sin Nombre virus, ${ }^{4}$ and 18 days (range 10-34 days) in a Chilean outbreak caused by the Andes virus. ${ }^{5}$

Our patient's disease course closely reflected the four phases of hantavirus cardiopulmonary syndrome described in the literature: the prodromal or febrile, cardiopulmonary, oliguric or diuresis, and convalescence phases. Fever, chills, myalgia, headaches and gastrointestinal symptoms characterize the prodromal or febrile phase. The cardiopulmonary phase occurs rapidly and is characterized by acute respiratory distress syndrome (ARDS), hemoconcentration, cardiovascular collapse and disseminated intravascular coagulation lasting from two to seven days. The oliguric or diuresis phase, with various degrees of acute renal failure, lasts between three and seven days, with subsequent recovery. ${ }^{6}$ The transition to the convalescent phase, with respiratory and cardiovascular recovery, can be as prompt as the onset of the condition.

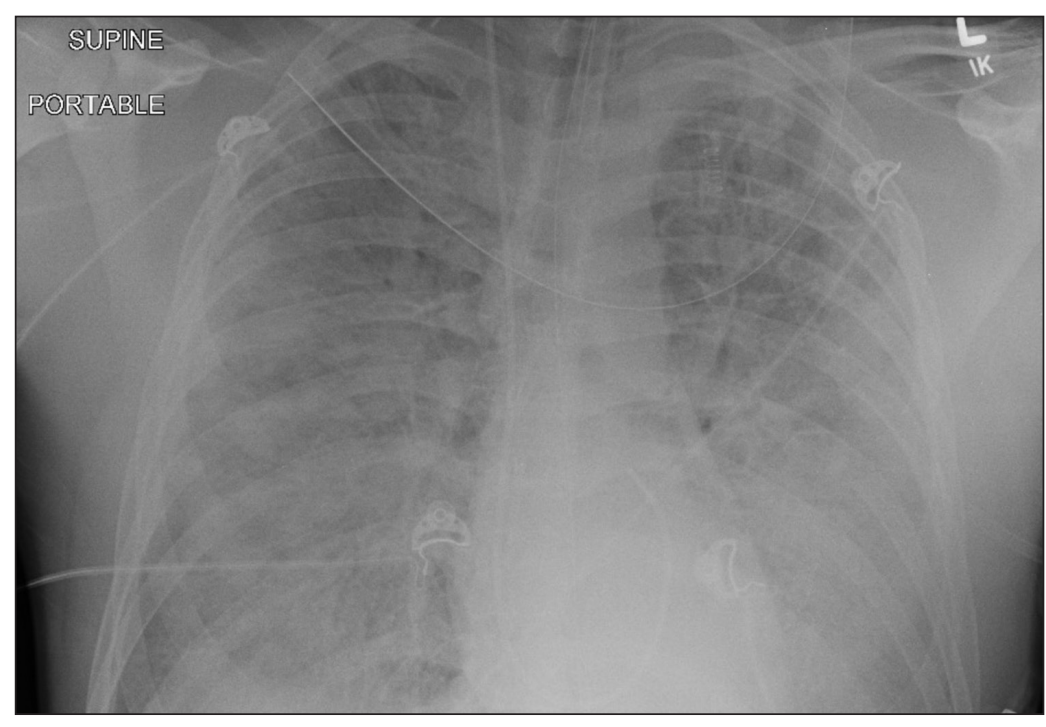

Figure 1: Anteroposterior radiograph of the chest of a 22-year-old man. Bilateral alveolar infiltrates, consistent with marked pulmonary edema, and bilateral pleural effusions can be seen. 


\section{Diagnosis}

Hantavirus cardiopulmonary syndrome should be suspected in patients who come from rural areas or with suspected exposure to rodents and who present with compatible symptoms. In the appropriate clinical context, the combination of pulmonary edema, thrombocytopenia, leukocytosis with left shift and hemoconcentration may help contribute to a presumptive diagnosis of Hantavirus infection. ${ }^{?}$ Suspected cases can be confirmed with serologic testing with IgM and IgG Hantavirus-specific antibodies, in addition to PCR analysis of serum and nasopharyngeal swabs. ${ }^{6}$ Antiviral IgM develops within two days; IgG develops within one week of initial symptom onset. Serum PCR analysis using appropriate primers become positive early in the pulmonary phase of the disease. Because viral RNA rapidly disappears from circulation, serology is the preferred diagnostic method. ${ }^{8}$

Centres without on-site testing can use clinical and laboratory markers for rapid presumptive diagnosis. ${ }^{8}$ The triad of simultaneous thrombocytopenia, left-shifted granulocytic cell line and immunoblasts on peripheral blood smear is highly suggestive of hantavirus cardiopulmonary syndrome and should trigger consideration for extracorporeal membrane oxygenation. ${ }^{7}$

\section{Treatment}

Treatment for hantavirus cardiopulmonary syndrome is supportive, with invasive ventilation, hemodynamic support, early use of vasopressors and cautious use of crystalloid. ${ }^{8}$ Ribavirin has been effective in treating hemorrhagic fever with renal syndrome, but its use is unproven in hantavirus cardiopulmonary syndrome. ${ }^{7}$ High-dose glucocorticoids are ineffective. ${ }^{8}$ Currently, there is no antiviral medication, immunotherapy or vaccine effective against the syndrome. In the last 20 years, VAECMO has come to the forefront as a supportive bridge to recovery in severe refractory hantavirus cardiopulmonary syndrome, as those who survive the acute cardiopulmonary phase recover quickly.

In 2011, Wernly and colleagues published a retrospective case series involving 51 patients with severe hantavirus cardiopulmonary syndrome supported with VA-ECMO. Patients had post-hoc serologic diagnosis of the syndrome and independent factors previously shown to be associated with $100 \%$ predicted mortality, including serum lactate greater than $4.0 \mathrm{mmol} / \mathrm{L}$, a $\mathrm{PaO}_{2} / \mathrm{FiO}_{2}$ ratio of less than 60 , refractory shock and cardiac arrest. Patients were given VA-ECMO and had a survival rate of $66.6 \%$. $^{9}$ This case series suggested that earlier introduction of VA-ECMO with anticipatory cannula insertion at the time of intubation was associated with improved survival $(80 \%$ v. $56 \%, p=$
0.048). ${ }^{9}$ Although there are no randomized controlled trials of VA-ECMO for hantavirus cardiopulmonary syndrome, suggested clinical criteria for starting VA-ECMO include presentation consistent with hantavirus cardiopulmonary syndrome, cardiac index of less than $2.0 \mathrm{~L} / \mathrm{min}$ per $\mathrm{m}^{2}$ and either refractory shock, a $\mathrm{PaO}_{2} / \mathrm{FiO}_{2}$ ratio of less than 60 or external cardiac massage. ${ }^{9}$

\section{Conclusion}

We report a Canadian adult patient presenting with severe, refractory hantavirus cardiopulmonary syndrome successfully treated with VAECMO support. Although the use of VA-ECMO for severe hantavirus cardiopulmonary syndrome has been described in the US, most Canadian provinces have little experience with Hantavirus infection. Given an increase in the incidence of Hantavirus in Canada, the potential for travelrelated exposures leading to presentations in nonendemic areas and the effectiveness of VAECMO in cases of rapid disease progression, physicians should be familiar with hantavirus cardiopulmonary syndrome so that urgent transfer to a centre capable of VA-ECMO can be arranged.

\section{References}

1. Padula PJ, Colavecchia SB, Martinez VP, et al. Genetic diversity, distribution, and serological features of hantavirus infection in five countries in South America. J Clin Microbiol 2000;38:3029-35.

2. Drebot MA, Jones S, Grolla A, et al. Hantavirus pulmonary syndrome in Canada: an overview of clinical features, diagnostics, epidemiology and prevention. Can Commun Dis Rep 2015;41:124-31.

3. Webster D, Lee B, Joffe A, et al. Cluster of cases of hantavirus pulmonary syndrome in Alberta, Canada. Am J Trop Med Hyg 2007;77:914-8.

4. Nunez JJ, Fritz CL, Knust B, et al. Hantavirus infections among overnight visitors to Yosemite National Park, California, USA, 2012. Emerg Infect Dis 2014;20:386-93.

5. Martinez-Valdebenito C, Calvo M, Vial C, et al. Person-to-person household and nosocomial transmission of Andes hantavirus, Southern Chile, 2011. Emerg Infect Dis 2014;20:1629-36.

6. Peters CJ, Khan AS. Hantavirus pulmonary syndrome: the new American hemorrhagic fever. Nephrol Dial Transplant 2002; 34:1224-31.

7. Mertz GJ, Hjelle B, Crowley M, et al. Diagnosis and treatment of new world hantavirus infections. Curr Opin Infect Dis 2006;19: 437-42.

8. Hjelle B. Hantavirus cardiopulmonary syndrome. UpToDate; 2008. Available: www.uptodate.com/contents/hantavirus-cardio pulmonary-syndrome (accessed 2016 June 1).

9. Wernly JA, Dietl CA, Tabe CE, et al. Extracorporeal membrane oxygenation support improves survival of patients with Hantavirus cardiopulmonary syndrome refractory to medical treatment. Eur J Cardiothoracic Surg 2011;40:1334-40.

Affiliation: Department of Medicine, McGill University Health Centre, Montréal, Que.

Contributors: Dr. Han Yao and Dr. Emily McDonald contributed to the design, redaction and revision of the manuscript, and to the care of the patient during his hospital stay. Both of the authors approved the final version of the manuscript to be published and agreed to act as guarantors of the results.

Acknowledgements: The authors thank Dr. Vivian Loo for the microbiologic diagnosis. The authors also thank Dr. Sheldon Magder and the McGill University Health Center Critical Care Department for the care of the patient during his stay in intensive care. 